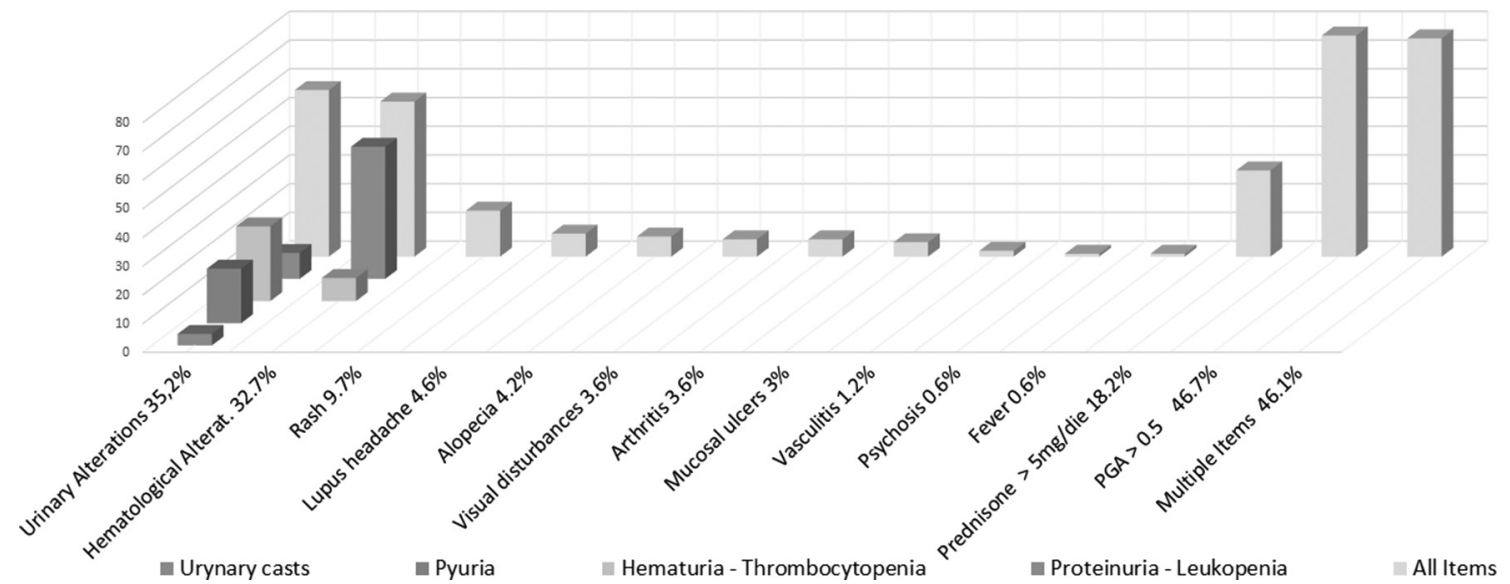

Abstract PS7:144 Figure 1

dose of corticosteroids $<5 \mathrm{mg}$ and/or immunosuppressants and/ or biologics drugs.'Clinical' remission was defined as the absence of any increase in corticosteroids dosage or any change in immunosuppressants.

Results 85SLE patients were enrolled (95\% female). 21\% of patients were in remission in all the 5 time-points, $23 \%$ never got into remission. $55 \%$ of patients satisfied DORIS criteria at least in one time-point. Mean duration of DORIS remission was 9 months.In 169 (40\%) visits there was a disagreement between DORIS and Clinical definition of Remission: a) in $2 \%$ remission according to DORIS but no clinical remission; b) $98 \%$ clinical remission but not according to DORIS.

The reasons for discordant results were: a) self-management of steroids dosage and precautionary increase of steroids in the suspect of a flare; b) cSLEDAI $>0$ in $74 \%$,PGA $>0.5$ in $47 \%$, daily prednisone $>5 \mathrm{mg}$ in $18 \%$. The cSLEDAI items that most contributed to the score were urinary and haematological alterations (figure 1).In 30 visits (16 patients) a clinical definition of remission was given despite a daily prednisone dose higher than $5 \mathrm{mg}$.

Conclusion Nearly $40 \%$ of the visits displayed a disagreement between 'clinical' and DORIS remission. This may be attributable mainly to a different approach in evaluating patients: longitudinal in clinical remission and cross-sectional by DORIS. As compared to 'clinical' remission, DORIS definition:

- may fail to recognise patients with a chronic stable steroid treatment at medium dosage, due to persistent low disease activity;

- is less sensitive because of PGA being used as a dichotomous variable with a low threshold;

- is likely to be scored different than zero because of urinary and haematological alterations.

\section{PS7:145 IL-34, NOT CSF-1, SIMILARLY MEDIATES RHEUMATOID AND LUPUS ARTHRITIS IN PATIENTS}

1J Weinmann-Menke, ${ }^{1} \mathrm{M}$ Meineck, ${ }^{2} \mathrm{~L}$ Cavagna, ${ }^{3} \mathrm{C}$ Brochausen, ${ }^{4} \mathrm{~V}$ Kelley, ${ }^{1} \mathrm{~A}$ Schwarting. ${ }^{1}$ Johannes Gutenberg University I. Department of Nephrology and Rheumatology, Mainz, Germany; ${ }^{2}$ Center of Rheuma-Pathology Division of Rheumatology University and IRCCS Policlinico S. Matteo Foundation, Pavia, Italy; ${ }^{3}$ Center of Rheuma-Pathology Medical Centre of the Johannes-Gutenberg University Mainz, Mainz, Germany; ${ }^{4}$ Laboratory of Molecular Autoimmune Disease, Renal Division, Department of Medicine, Brigham and Womens Hospital, Boston, USA

10.1136/lupus-2018-abstract.188
While Myeloid cells are abundant in lupus arthritis (LA) and rheumatoid arthritis (RA), based on clinical presentation LA and RA are considered distinct diseases. Although inflammatory arthritis is common in patients with lupus, the pivotal mechanisms leading to joint damage have not been investigated. We tested the hypothesis that IL-34, but not CSF-1, is a predictive biomarker that is integral in perpetuating synovial destructive inflammation in both LA and RA. We report the novel findings that:

i. using longitudinally tracked patients, IL-34, not CSF-1, is a clinical predictive biomarker for both LA and RA; and

ii. IL-34 is more robustly expressed in the synovial tissue, cells and fluid compared to CSF-1 in both LA and RA.

Probing into the IL-34 dependent mechanisms in vitro we find that:

i. IL-34 promotes synovial hyperplasia more robustly than CSF-1, and increases chemokines that recruit neutrophils and monocytes (Mo) into the synovium in LA and RA;

ii. Mo and neutrophils stimulated by IL-34, via cell contact and/or released mediators, such as ROS, destroy synovial cells;

iii. signalling via the two IL-34 receptors, cFMS and proteintyrosine phosphatase (PTPRZ), promote IL-34 and CSF-1 mediated synovial cell hyperplasia and cytotoxicity.

Taken together, IL-34-dependent mechanisms are pivotal and similar in mediating LA and RA. Moreover, tracking serum IL-34 is a reliable biomarker for managing the individualised treatment of patients with both LA and RA.

\section{PS7:146 INVESTIGATION OF CHRONIC ORGAN DAMAGE AND DISEASE OUTCOME IN HUNGARIAN LUPUS PATIENTS}

T Tarr, G Papp, N Nagy, M Zeher. University of Debrecen, Debrecen, Hungary

\subsection{6/lupus-2018-abstract.189}

Introduction/objectives Long-term survival of patients with systemic lupus erythematosus (SLE) improved worldwide, thus prevention of cumulative organ damage became a major goal in disease management. The aim of our study was to investigate the chronic organ damages and their influence on disease outcome in SLE.

Method We evaluated clinical conditions, laboratory findings and medications of 357 consecutive SLE patients, and assessed 